\title{
Categorical Cross-Recurrence Quantification Analysis Applied to Communicative Interaction during Ainsworth's Strange Situation
}

\author{
Danitza Lira-Palma, Karolyn González-Rosales, Ramón D. Castillo (D), \\ Rosario Spencer, and Andrés Fresno \\ Centro de Investigación en Ciencias Cognitivas, Facultad de Psicología, Universidad de Talca, CP 3460000, Chile \\ Correspondence should be addressed to Ramón D. Castillo; racastillo@utalca.cl
}

Received 16 May 2018; Revised 21 August 2018; Accepted 26 September 2018; Published 1 November 2018

Guest Editor: Ralf Cox

Copyright @ 2018 Danitza Lira-Palma et al. This is an open access article distributed under the Creative Commons Attribution License, which permits unrestricted use, distribution, and reproduction in any medium, provided the original work is properly cited.

\begin{abstract}
The goal of this study was to characterize the degree of structuring of verbal and motor behaviours, unfolded during the application of an procedure called the Strange Situation. This procedure is used for assessing children's attachment quality during early stages of their development. Many studies have demonstrated that communicative interactions share features with complex dynamic systems. In such studies, estimations of degree of structure have been used to characterize the system's synchronization. Thus, assuming that processes of communicative interaction occur in the Strange Situation procedure, it was expected to find traces of synchronization. The metrics were estimated through a Categorical Cross-Recurrence Quantification Analysis applied to the behaviours of individuals and dyads. Two applications of the Strange Situation were implemented and recorded. Verbal and motor interactions among children, caregivers, and strangers were transcribed, categorized, and organized as time series. From each time series of original behaviours, randomized time series were created. Measures of recurrence extracted from Recurrent Plots, such as determinism, entropy, maximum line, laminarity, and trapping time, were calculated. Original and randomized time series were compared in terms of these measures. Results indicated that communicative interaction during the Strange Situation had a structure that mimics properties observed in social interactions where synchronization emerges. In our case, verbal behaviours were more prone to synchronization than motor behaviours, in both individuals and dyads, even though this pattern was more salient among caregivers and strangers than children. The relevance of having measures that can capture synchronization during the administration of the Strange Situation is discussed. Our preliminary findings allow us to point out that the application of RQA and C-RQA to the Strange Situation could not only contribute to methodology, but also contribute to emphasizing the role of coupling in communicative interaction generated by the application of this procedure to measure attachment patterns.
\end{abstract}

\section{Introduction}

Human communicative interaction is a phenomenon that behaves as a complex dynamic system [1-3]. The reason for the complexity observed in communicative interaction is because the number of components and relations between them increases to such an extent that a new phenomenon emerges, and this phenomenon cannot be explained by the constitutive components [2]. Complex dynamic systems, whether physical, chemical, biological, or social, share distinctive properties, among which synchronization is relevant. In broad terms, synchronization refers to the activity of two or more components at the same time or rate. In other words, this process occurs when two or more systems recurrently share a trajectory over a certain period [4].

Synchronization has shed light on the understanding of the development, permanence, and fluctuation of a complex system. Research conducted under the dynamic system approach proposes that human communicative behaviour-at the intra- and interindividual levelsproduces synchronization and coupling [2]. Concordantly, findings indicate that some social-communicative interactions have characteristics of a complex dynamic system, where synchronization is a pivotal attribute [5]. 
Evidence of synchronization of intraindividual behaviours has been found in a series of perceptual [6-8], motor [912], and simple decision tasks [13-16]. Signs of synchronization between individuals have been observed in cooperative and noncooperative situations; and traces of coupling have been observed in linguistic and nonlinguistic interactions. Taking these results into account, it has been proposed that synchronization can be modulated by contextual factors [17]. For example, in a social context with negative valence, lower levels of synchrony were detected among the participants. Vink et al. [18] systematized scientific research and reported greater levels of synchronization between dyads when selfreports of rapport were described in terms of more intensity. Hove and Risen [19] found levels of sympathy were positively associated with levels of synchronization between participants. They also observed that when a partner (confederate) came later to the experiment to perform a task there were low levels of interpersonal synchrony between participant and confederate.

Complementarily, other studies have identified that coupling dynamics also vary depending on the conversational context. When a person speaks to a listener who is located somewhere else, better levels of understanding were observed with a delay of two seconds [20]. With this delay, it was also found that gazes exhibit synchronization and coupling. On the other hand, when two persons speak face to face, in real time, alternating the role of speaker and listener, the optimal coupling time is reduced to zero seconds [21].

As a result of communicative interaction, synchronization plays an important role for social development [22]. It has been suggested that synchrony in mother-child interactions is not only significant for language acquisition but also significant for the development of social relationships and intersubjectivity. Along the same line, Stern [23] found that a lack of synchronization between parents and their children could affect the latter's behaviour and affective states.

A longitudinal study developed by Siller and Sigman [24], with parents of children with autism, found that the communication between caregivers and children was predicted by the degree of synchronization in the interaction. Tunçgenç and Fawcett [25] conducted two studies in which children who were 9 months old and 12 months old were located in social and nonsocial contexts. They found that 9-monthold babies showed preference for objects in synchronous movement, regardless of whether the object was in a social context or not. However, 12-month-old infants showed a preference only for stimuli in a social context that moved synchronously with respect to them. Thus, synchronization of the movements was an important factor to guide the social preferences of babies [25].

In sum, human communicative behaviour is considered a complex dynamic system, where synchronization is a relevant attribute. Signs of synchronization have been detected in various types of behaviour and cognitive processes, in both individuals and groups. There is evidence that levels of synchronization can affect and be affected by the initial conditions of the environment or by the affective-emotional state of the individuals. Furthermore, based on the aforementioned empirical background, it is possible to conjecture that the presence of synchronization in behaviour is an early marker of healthy development and that synchronization enables predicting the adaptive behaviour of humans in the early stages of their development.

The Theory of Attachment was proposed Bowlby [26] to understand how these early communicative behaviours and the degree of social adaptive behaviour are configured by the caregiver-child interaction. This theory explains how the first relationships of children with their caregiver are formed, based on the concept of attachment, which is described as "(...) the process by means of which children establish and maintain a special relationship with another individual who is considered better able to face the world" [27, p. 40]. Patterns of attachment are the result of instinctive responses for the protection and survival of the child. This process is considered as the bridge of early development and later development of social relations. Thus, the bond of attachment could be considered as the relationship established between babies and their caregivers, which influences their development and subsequent well-being.

There is an increasing amount of research about attachment in the early stages of development, personality development $[28,29]$, social adjustment $[23,24]$, as well as the development of psychopathology [30]. The collected background information indicates that the configuration of a particular attachment pattern is the result of an interaction between the natural dispositions of the child and the communicative and bonding patterns that caregivers manifest in a critical period [21]. Disruptions in the period in which this early bond is configured can have negative effects, of great impact, on the lives of individuals.

Patterns of attachment are observed under an experimental procedure called the Strange Situation (SS) [31]. The SS, developed by Ainsworth and colleagues, is the gold standard method to assess the quality of infant-caregiver attachment bond [32]. By proposing a mildly to moderately stressful experience for the infant, this laboratory assessment procedure activates the infant's attachment behaviour addressed to the attachment figure (the caregiver). The increase of the infant's stress activates the infant's attachment behavioural system. These attachment behaviours reveal how the infant organizes his/her expectations regarding the availability of the adult and how he/she can use the caregiver in order to return to calm. Once the infant is reassured, the attachment behavioural system is deactivated, and the exploration behavioural system is activated, evidencing the balance between attachment and exploration [33]. Thus, this procedure aims to identify patterns of attachment between the child and his or her primary caregiver in a laboratory situation where the child's stress is gradually increased by the presence of a stranger and two brief separations from the caregiver. The procedure is composed of eight episodes with a duration of three minutes each [30]. Episodes 5 and 8 are the moments in which the caregiver meets with the child, after having been separated in the previous episodes. The child's reactions are scored and categorized according to four criteria: Proximity and contact seeking; contact maintaining; avoidance of proximity and contact; and resistance to contact and comforting. Finally, according to Ainsworth 
[31] and Ainsworth et al. [32], children's reactions under the Strange Situation are classified into four patterns of attachment: (B) Secure: This pattern describes a child who uses the caregiver as a safe base for exploration and can manifest stress behaviour during separation. During the meeting, the child actively seeks caregiver contact through behaviours such as smiles, vocalizations, gestures or physical approach. (A) Insecure Avoidant: This pattern describes a child that shows exploration behaviours but displays few affective behaviours or rarely uses the caregiver as a safe base. During the separation, the child shows slight or no sign of stress. At the meeting, the child tends to avoid contact with the caregiver, such as avoiding gaze and physical contact or focusing attention on toys rather than the caregiver. (C) Resistant Insecure: This pattern depicts a child that during the separation seems extremely stressed. At the meeting, the child usually alternates contact and seeking of the caregiver with signs of rejection, even tantrums towards the caregiver. The child can also be very passive or show behaviours that denote anger. (D) Disorganized Insecure: In this pattern, the child expresses a series of contradictory or incomplete behaviours that would denote a lack of structure, such as interrupted movements, stereotyped movements, freezing/stilling, indicators of fear/apprehension, disorientation, and confusion towards the caregiver [21].

From a dynamic complex system perspective, these attachment patterns should interact with other variables to give rise to a particular type of interaction $[2,8]$. Furthermore, these patterns of attachment would also be an integral part of the synchronization with other people. Generally, children's attachment would be an important ingredient for synchronization of behaviours observed in social interactions with adults [11, 14, 34]. Thus, as the SS is an experimental protocol that promotes social-communicative interactions, and social interactions have shown attributes of complex dynamic systems, it was hypothesized that traces of synchronization between dyadic interactions of caregivers, strangers and children could be found.

Research in human communicative interactions has shown that synchronization is nonstationary; it experiences fluctuations and transitions $[35,36]$. From a traditional perspective, these aspects are usually controlled or avoided because they add error to the results. However, from a dynamic system approach these aspects, rather than avoided, must be incorporated due to their informative nature. Abrupt changes in postures, introduction and changes of topics, and breaks in the continuum of the conversation, among other factors, could be indicative of qualitative shifts in the mental states of individuals in response to a particular situation [35, 37]. These behaviours are not isolated but rather chained, and they express a pattern that can be identified when are repeated over time. We hypothesized that Ainsworth's Strange Situation, even though it is a highly standardized protocol, has communicative aspects that show traces of relative synchronization between the actors. These signs of synchronization should be identified whether the behaviour is analysed over time, incorporating all aspects of the dynamics, such as fluctuations, transitions and stationarity.
Researchers in the field of dynamic systems have developed a series of techniques and parameters to study synchronization without abandoning its critical aspects, such as nonstationarity, fluctuations, and transitions. One technique that has proven to be useful in the analysis of system synchronization is the Recurrence Quantification Analysis (RQA) [38]. RQA is a multidimensional nonlinear method used to discover attractors from tenuous correlations and subtle repetitive patterns in a time series where the data are noisy, irregular, and with many factors or dimensions affecting their configuration $[8,39,40]$. RQA does not require additional treatment or assumptions about data distribution or size, and it can be applied to both linear and nonlinear variables $[8,39,41]$. Measures extracted by mean of RQA are estimated from recurrence plots (RPs). As depicted in Figure 1, the RP is a graphical representation of a matrix of recurrence that highlights aspects that cannot be detected in the original data set. In formal terms, the RP is an autocorrelation of $\mathrm{x}_{(\mathrm{t})}$ with $\mathrm{x}_{(\mathrm{i})}$ through the abscissa and $\mathrm{x}_{(\mathrm{j})}$ through the ordinate. Only points that satisfy the condition $\mathrm{x}_{(\mathrm{i})}=\mathrm{x}_{(\mathrm{j})}$ are plotted [12, $41,42]$. From a RP, several quantitative and reliable measures can be estimated, such as the percentage of recurrence that quantifies the proportion of recurrent points that fall within the recurrent plot with a specified radius. The percentage of determinism quantifies the degree of randomness based on the proportion of recurrent points that form a diagonal line, called identity line [43]. Determinism allows knowing if future states of the system are determined by their previous states. Periodic signals can produce long diagonal lines; chaotic signals can generate short diagonal lines, and, finally, stochastic signals cannot generate any diagonal line at all. Entropy represents the uncertainty based on Shannon's entropy, which identifies the degree of disorder expressed by a system. This measure is calculated from the lengths of all diagonal lines that are organized in a histogram according to their distribution. For simple periodic systems, in which all diagonal lines have equal length, the expected entropy is equal to zero. The maximum line represents the length of the longest diagonal line on the RP when the diagonal line of identity has been excluded. Hence, it is a measure of system stability. If the length is shorter, the signal is chaotic, and if the length is larger, the signal is more stable [8].

Cross-Recurrence Quantification Analysis (C-RQA) is used with signals coming from two interacting systems [4450]. C-RQA, like RQA, quantifies coordinative patterns based on an analysis of the sequence of behaviours performed in real time [40, 41]. Figure 1 shows RPs with interesting features, which can be quantified in various ways [38]. One way is to focus on the diagonal line structures, because they depict a sequence of iterations. When the focus is on vertical lines, two additional measures can be estimated that are considered more informative in terms of the structure of two interacting signals: Percentage of laminarity that represents the proportion of recurrence points that form vertical lines. The laminarity percentage is similar to that of determinism, except that it depicts the proportion of recurrent points comprising vertical line structures rather than diagonal. Finally, there is another measure called trapping time, which represents the mean length of vertical lines. 


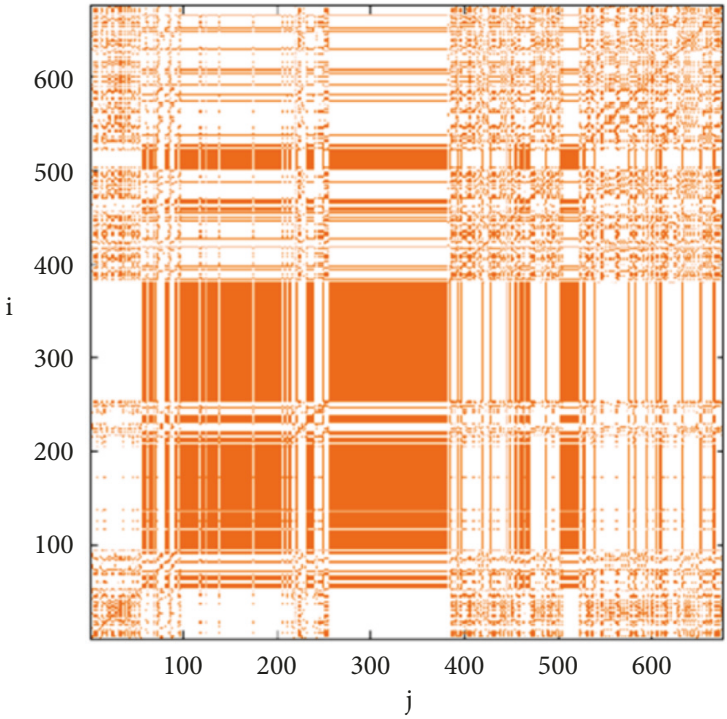

(a)

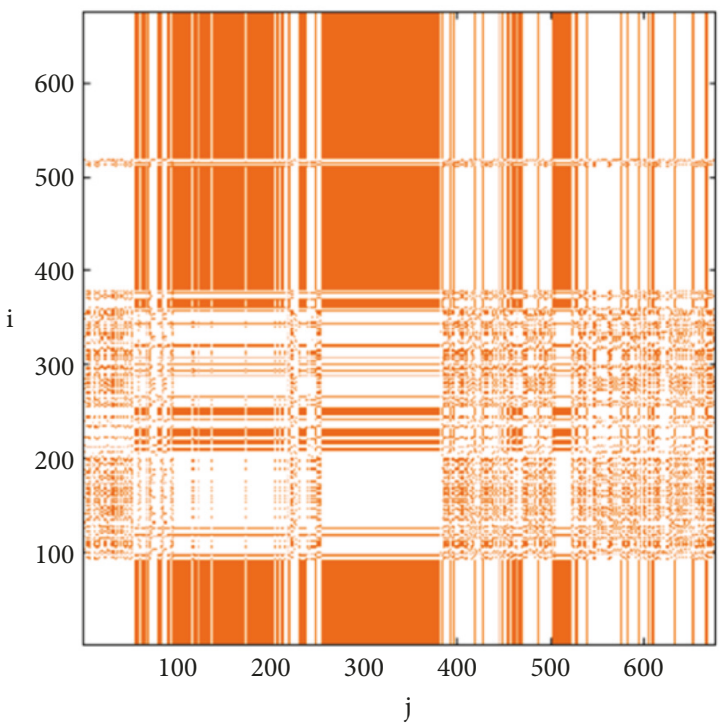

(c)

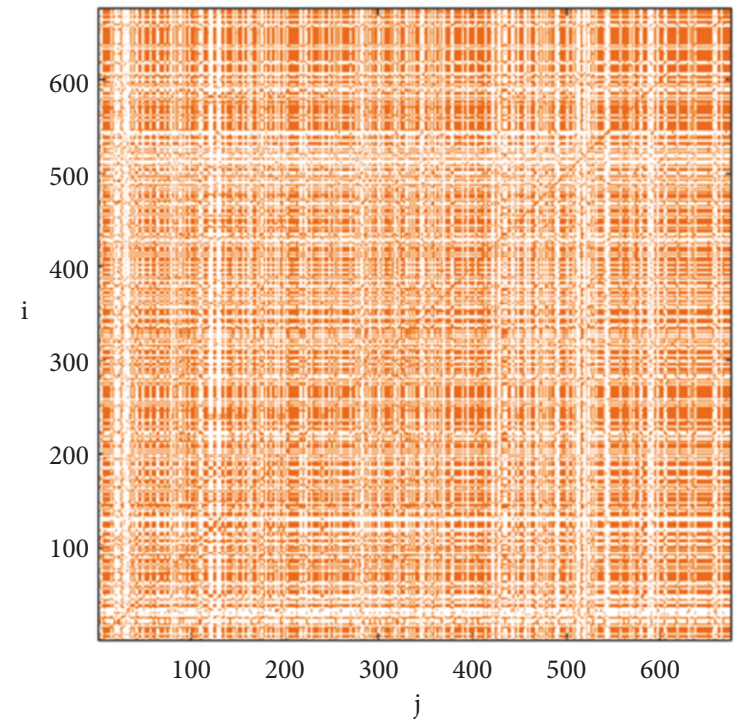

(b)

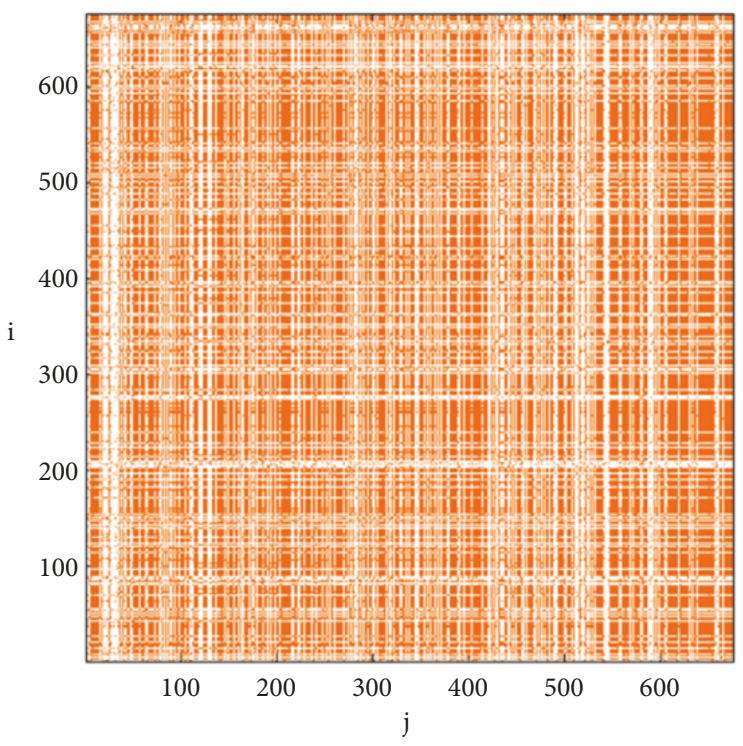

(d)

Figure 1: Recurrence plots (RP) of verbal expressions and grammar coming from an individual (Panels (a) and (b)) and dyads (Panels (c) and (d)). Panel (a) shows a RP with a delay of 1 and an embedded dimension equal to 1 , in which it is possible to observe a diagonal line and coloured squares that show repetitions-in speech-of certain grammatical structures (e.g., verb, pronoun, adjective, article, adverb, among others) while the individual was interacting communicatively. Panel (b) shows a RP in which the original verbal expressions were randomized (reshuffled). Randomization destroyed the sequences and, therefore, the coherence with which the behaviours was appearing while the interaction was taking place. Thus, the RP represented by Panel (b) shows a random pattern of behaviours, in which predictability is very low, there is maximum entropy, and there is no system stability at all. Panel (c) shows a RP generated from a dyadic interaction in which C-RQA was applied. This RP and its randomized version (Panel (d)) have a characteristic that differentiates them from the RPs in panels (a) and (b). This is because the diagonal identity line is not present. Being two systems that interact, the diagonal line of identity tends to disappear.

RQA and C-RQA have been applied to detect recurring features and patterns of complex dynamical systems, which result from one or two signals interacting over time [38, $43,48]$. In psychology, such signals can be fluctuations in gait, postural changes, eye movements, or informational patterns, such as syntactic structures or words exchanged by two persons during a communicative interaction. In fact, there is a large corpus of evidence in which these nonlinear techniques have been used to analyse postural fluctuations [42], conversational interaction between caregiver and child $[34,51,52]$, coupling of time series of verbalizations and gestures [50], and reading comprehension [53]. Additionally, 
through these analyses, interpersonal coordination has been characterized in terms of coupling of eye movements [42], body movements [41, 54], child behaviour and sleep [55], patterns of aggression [29], minimal and effective forms of coordination during the dialogue [4], and early language development [46, 47].

Even when RQA and C-RQA have been used in diverse contexts of communicative interaction, until now no research has been aimed at studying the communicative interaction of standardized routines with children, such as the Strange Situation. The implementation of Ainsworth's Strange Situation is organized in a very systematic and interesting way, and new techniques can help researchers to examine its temporal organization. Even though the main goal is to define the kind of attachment pattern of the infant, it is equally important to know how verbal expressions and motor behaviours are unfolded, and whether the structure observed in such variables can be used to estimate how traces of synchronization emerge among the individuals present during the evaluation.

Based on this background, our research aimed at analysing the unfolded verbal and motor behaviours among the participants of the Strange Situation (caregiver, child, and stranger), and to estimate, in reliable terms, the structure of these behaviours. The questions that we intend to answer are: What are the structural indicators of these behaviours that allow establishing traces of synchronization between the actors of the Strange Situation? What values do recurrence measures adopt in the behaviours of each individual and dyad (caregiver-child, stranger-child, and caregiver-stranger)?

\section{Materials and Methods}

Before implementing this research, the protocol was reviewed and approved by the University's Institutional Review Board (IRB \# 1161533 and IRB \# 1130773).

2.1. Participants. Two female infants, 14 - and-18-month old, with their respective biological mothers, were recruited for this research. Both mothers were married and had completed undergraduate studies, and, according to their income, they were classified as middle class. The "strangers" were two clinical psychologists, 25 and 30 years old each. Both specialists were trained and certified in the use of the protocol of the Strange Situation. Before carrying out the study, each mother-caregiver read and signed the informed consent that was explained in detail by the researchers in charge of the study. Clinical psychologists were randomly assigned as evaluators when the procedure of the Strange Situation was administered. The sessions were recorded in a Gesell room equipped with high-resolution cameras and microphones.

\subsection{Procedure}

(1) Evaluation with Strange Situation Protocol. Following the protocol proposed by Ainsworth and collaborators [32], the assessment took about twenty minutes, organized in eight episodes that combined the separation and the reunion of the infant and the caregiver. The episodes lasted three minutes each. In the first episode, the caregiver enters the room with the child. In the second episode, the caregiver takes a seat while the child can interact with the toys. In this phase, the caregiver can interact with the child if the child requests it. In the third episode, the stranger enters the room, and takes a seat without interacting with the child or caregiver for a minute. The stranger then talks to the caregiver for a minute and then plays with the child for one minute. Episode four begins when the caregiver leaves the room, while the stranger stays with the child in the room. If the child is distressed, the stranger can comfort the child. In the fifth episode, the caregiver returns to the room and the stranger leaves. The caregiver knocks on the door before entering and says the name of the child. After waiting for a moment, she is free to respond to the child. She must then make the child interested in the toys and sit down again. In the sixth episode, the child is left alone in the room. In episode seven, the stranger enters the room. If the child is in distress, the stranger can comfort the child. In episode eight, the mother enters and the stranger leaves the room. The caregiver can behave in the same way as in the previous meeting. If the child is very stressed by the separations, these periods can have a shorter duration (30 seconds). The behaviours of the child observed during the two meetings (episodes 5 and 8) are coded in four scales (Proximity and contact seeking; contact maintaining; avoidance of proximity and contact; and resistance to contact and comforting). Based on these scores and taking into account the behaviour of the child throughout the procedure, the child is classified in the category of secure attachment (B), insecure avoidant (A), or resistant insecure (C). If, during the episodes that provided contact with the mother, the child presented disorganized behaviours that disrupted the organization of their attachment relationship, the classification applied is disorganized insecure attachment (D).

\section{(2) Analysis and Categorization of Verbal and Motor} Behaviours

(2.1) Words Were Labelled with a Number by Using a Text Converter. Regardless of the language, this text converter assigns a number that is characteristic and unique to each word (http://cognaction.org/rick/ati/). The procedure was repeated twice, comparing the numbers assigned to the word sequences. The reliability in assigning numbers to each word was perfect, with a Kappa value equal to one.

(2.2) The Text Converter Does Not Analyse Verbal Expressions, Grammar in Spanish, or Any Other Type of Communicative Behaviour. The coding of these variables was implemented manually. Verbal expressions and grammar were grouped in 43 descriptors. Body movements were grouped in 59 descriptors. The labels and their descriptions are provided in Tables 1 and 2. Two trained researchers, who did not participate in the application of the Strange Situation procedure, analysed the videos and categorized the behaviours of caregivers, strangers and children. These researchers were blind to the children's attachment pattern. Verbal expressions, babblings 
TABLE 1

\begin{tabular}{lc}
\hline Code & Verbal expressions and Grammar \\
\hline 1 & Silence \\
2 & Inarticulate language (Crying) \\
3 & Inarticulate language (babbling) \\
4 & Inarticulate language (Scream) \\
5 & Article \\
6 & Pronoun \\
7 & Noun \\
8 & Verb \\
9 & Adjective \\
10 & Adverb \\
11 & Conjunction \\
12 & Preposition \\
13 & Interjection \\
14 & Contraction \\
15 & Adverbial phrase \\
16 & Own name \\
21 & Crying \\
22 & Crying, complaint \\
23 & Much stronger crying \\
31 & Babbles sound “Aa" \\
32 & Babbles sound "Ee" \\
33 & Babbles sound "Ii”" \\
34 & Babbles sound "Oo" \\
41 & Shouts “Aa" \\
42 & Shouts "Ee" \\
43 & Shout s"Ii” \\
\hline &
\end{tabular}

and periods of silence were transcribed and coded. A similar transcription and coding was done for body movements and gestures, where each behaviour was registered according to the moment it appeared. The interrater reliability between these two researchers was estimated with a Kappa Coefficient (Table 3) during three segments. The Kappa values were highly reliable, fluctuating between 0.71 and 1.0.

Each video was divided into a discrete number of events. There were 676 events for Video 1 and 400 for Video 2. For each participant in the Strange Situation (child, caregiver, and stranger) verbal expressions and grammar, as well as body movements were maintained in the order in which they appeared. For each participant, three files were extracted with the original time series of the three types of variables. For each variable, randomized time series were generated. Thus, for each child, caregiver, and stranger there were six files containing the three original series and their respective randomized series.

From a dynamic perspective it has been proposed that original series of behaviour form regular patterns, which have certain properties different from the same randomized series [6-10, 12-16]. A strategy to account for the dynamic character of the behaviour has been to compare the original series with the same randomized series [53]. This strategy has been implemented with fractal techniques, such as Detrended Fluctuation Analysis, Standardized Dispersion Analysis, and
Spectral Analysis [56-58], and also applied with complexity measures such as RQA and C-RQA [13, 41]. Even when there are opinions contrary to this strategy and its assumptions $[59,60]$, some researchers had shown that this strategy is convergent with other procedures that have proven to be robust in demonstrating the dynamic and complex nature of cognitive and behavioural processes [61].

Figure 2 depicts an example of this manipulation in which verbal expressions were categorized. For the original series (Panel (a)), 45 consecutive events were selected in which caregiver and stranger were interacting (between event 205 and event 251). The numbers represent certain types of words (verbs, nouns, articles, pronouns, adverbs, among others). For the randomized series (Panel (b)), the words that were located between events 205 and 251 were selected, Differences between the original and randomized series can be visually detected. However, it is difficult to detect differences in series of 676 or 400 events. The same procedure was implemented with child-caregiver and child-stranger dyads. The complete string of verbal expressions and grammar was made up of 676 events for Video 1 and 400 events for Video 2. For individuals and dyads, original and randomized series were analysed by means of categorical RQA and C-RQA, respectively. From these analyses, measures of synchronization, such as determinism, maximum line, entropy, laminarity, and trapping time were estimated. Analyses were applied on original and randomized series, in order to be compared. Assuming that all communicative interactions have a dynamic structure in natural conditions, randomization should annihilate such structure. If the original and randomized series have the same value of determinism, entropy, laminarity, maximum line, and trapping time, it can be concluded that such a system has no coupling or synchronization.

Following the guidance of Dale and colleagues [51] for the implementation of RQA and C-RQA with categorical data, recurrence measures were estimated with one embedded dimension, and with delays of one lag. Unlike the analysis with continuous variables, in this case radius and delay were not estimated.

\section{Results and Discussion}

3.1. Categorical Recurrence Quantification Analysis for Individuals in Videos 1 and 2. The results for children, caregivers, and strangers are summarized in Table 4. It is possible to observe that the randomized and original series had the same level of recurrence. These results were expected, considering that randomized series were generated from the same data than the original data. Thus, the events that are part of the recurrence were organized in different order, but they are the same. Randomization breaks down the original structure, mainly affecting measures of determinism, entropy, maximum line, and laminarity. When the original structure of words, verbal expressions and grammar, and body movement was shuffled, a decrease in determinism and laminarity (lower predictability) and an increase in entropy (lower values of entropy in this case) were expected. These results, as predicted, were clearly observed in the words, 
TABle 2

\begin{tabular}{|c|c|}
\hline Code & Body Movements \\
\hline 1 & Silence \\
\hline 2 & Takes toy 1 \\
\hline 3 & Takes toy 2 \\
\hline 4 & Takes toy 3 \\
\hline 5 & Takes toy 4 \\
\hline 6 & Takes the paper \\
\hline 7 & Gets up off the floor (or the chair) \\
\hline 8 & Lightens up \\
\hline 9 & Moves hands \\
\hline 10 & Moves arms \\
\hline 11 & Moves head towards the girl \\
\hline 12 & Smiles \\
\hline 13 & Moves head towards the stranger \\
\hline 14 & Bends \\
\hline 15 & Moves head affirmatively (yes) \\
\hline 16 & Takes the girl's hand \\
\hline 17 & Takes the girl \\
\hline 18 & Walks \\
\hline 19 & Exits the room \\
\hline 20 & Enters the room \\
\hline 21 & Kisses the girl \\
\hline 22 & Sits the girl on the floor \\
\hline 23 & Touches the girl's head \\
\hline 24 & Hugs the girl \\
\hline 25 & Takes toys 1 and 3 \\
\hline 26 & Plays with toys 1 and 3 \\
\hline 27 & Drops toy or paper \\
\hline 28 & Points forward \\
\hline 29 & Aims towards toy 3 \\
\hline 30 & Moves the girl \\
\hline 31 & Sings \\
\hline 32 & Touches the girl \\
\hline 33 & Aims for the chair \\
\hline 34 & Moves toy 1 \\
\hline 35 & Moves toy 2 \\
\hline 36 & Moves toy 3 \\
\hline 37 & Bites toy 1 \\
\hline 38 & Bites toy 3 \\
\hline 39 & Hits toy 1 \\
\hline 40 & Hits toy 3 \\
\hline 41 & Drops toy 1 \\
\hline 42 & Drops toy 2 \\
\hline 43 & Drops toy 3 \\
\hline 44 & Picks up toy 3 \\
\hline 45 & Moves torso forward \\
\hline 46 & Moves torso towards the mother \\
\hline 47 & Crawls towards the mother \\
\hline 48 & Raises hands to the mother \\
\hline 49 & Moves torso towards stranger \\
\hline 50 & Hugs the mother \\
\hline 51 & Takes the mother's hand \\
\hline
\end{tabular}


TABLe 2: Continued.

\begin{tabular}{lr}
\hline Code & Body Movements \\
\hline 52 & Touches toy 2 \\
53 & Moves leg \\
54 & Crawls \\
55 & Touches toy 4 \\
56 & Plays with toys 1 and 4 \\
57 & Plays with toy 4 \\
58 & Aims for toy 3 \\
59 & Takes toys 1 and 3 \\
\hline
\end{tabular}

TABLE 3: Kappa values of interrater reliability.

\begin{tabular}{|c|c|c|c|c|c|c|c|c|c|}
\hline & \multicolumn{3}{|c|}{$\mathrm{Ca}$} & \multicolumn{3}{|c|}{$\mathrm{Ch}$} & \multicolumn{3}{|c|}{ St } \\
\hline & Segment 1 & Segment 2 & Segment 3 & Segment 1 & Segment 2 & Segment 3 & Segment 1 & Segment 2 & Segment 3 \\
\hline \multicolumn{10}{|c|}{ Verbal Expressions and Grammar } \\
\hline Video 1 & $1^{*}$ & $1^{*}$ & $.99^{*}$ & $1^{*}$ & $1^{*}$ & $1^{*}$ & $.98^{*}$ & $1^{*}$ & $1^{*}$ \\
\hline Video 2 & $1^{*}$ & $1^{*}$ & $1^{*}$ & $1^{*}$ & $1^{*}$ & $.94^{*}$ & $.98^{*}$ & $1^{*}$ & $1^{*}$ \\
\hline \multicolumn{10}{|c|}{ Body Movements } \\
\hline Video 1 & $.91^{*}$ & $1^{*}$ & $1^{*}$ & $.91^{*}$ & $1^{*}$ & $1^{*}$ & $1^{*}$ & $1^{*}$ & $1^{*}$ \\
\hline Video 2 & $1^{*}$ & $1^{*}$ & $.97^{*}$ & $1^{*}$ & $1^{*}$ & $.87^{*}$ & $.71^{*}$ & $1^{*}$ & $1^{*}$ \\
\hline
\end{tabular}

Note: $\mathrm{Ch}=$ child; $\mathrm{Ca}=$ caregiver; $\mathrm{St}=$ stranger $; ~^{*} p<.0001$.

TABLE 4: RQA measures for individuals in Videos 1 and 2.

\begin{tabular}{|c|c|c|c|c|c|c|c|c|c|c|c|c|}
\hline & \multirow[b]{2}{*}{ Variables } & \multirow[b]{2}{*}{ Unit } & \multicolumn{2}{|c|}{ Recurrence } & \multicolumn{2}{|c|}{ Determinism } & \multicolumn{2}{|c|}{ Entropy } & \multicolumn{2}{|c|}{ Maximum Line } & \multicolumn{2}{|c|}{ Laminarity } \\
\hline & & & Orig & Ran & Orig & Ran & Orig & Ran & Orig & Ran & Orig & Ran \\
\hline \multirow{9}{*}{ Video 1} & \multirow{6}{*}{ Verbal Expressions and Grammar } & $\mathrm{Ch}$ & 75.51 & 75.51 & 92.13 & 92.2 & 3.49 & 3.12 & 130 & 60 & 0.98 & 0.99 \\
\hline & & $\mathrm{Ca}$ & 51.41 & 51.41 & 95.64 & 77.43 & 3.96 & 1.9 & 130 & 6 & 0.92 & 0.72 \\
\hline & & St & 19.51 & 19.51 & 88.64 & 33.72 & 4.02 & 0.71 & 153 & 11 & 0.97 & 0.82 \\
\hline & & $\mathrm{Ch}$ & 75.22 & 75.22 & 92.61 & 94.44 & 3.39 & 3.19 & 69 & 43 & 0.98 & 0.99 \\
\hline & & $\mathrm{Ca}$ & 53.78 & 53.78 & 96.53 & 80.12 & 3.73 & 2.24 & 126 & 10 & 0.82 & 0.63 \\
\hline & & St & 24.65 & 24.65 & 80.6 & 38.23 & 3.25 & 0.88 & 155 & 13 & 0.93 & 0.84 \\
\hline & \multirow{3}{*}{ Body Movements } & $\mathrm{Ch}$ & 53.41 & 53.41 & 80.2 & 78.32 & 2.22 & 1.9 & 10 & 10 & 0.86 & 0.86 \\
\hline & & $\mathrm{Ca}$ & 43.39 & 43.39 & 73.39 & 68.79 & 2.71 & 1.55 & 37 & 8 & 0.75 & 0.81 \\
\hline & & St & 57.44 & 57.44 & 84.41 & 83.95 & 3.42 & 2.33 & 57 & 26 & 0.94 & 0.96 \\
\hline \multirow{9}{*}{ Video 2} & \multirow{6}{*}{ Verbal Expressions and Grammar } & $\mathrm{Ch}$ & 80.84 & 80.84 & 95.31 & 96.04 & 4.23 & 3.58 & 173 & 25 & 0.96 & 0.97 \\
\hline & & $\mathrm{Ca}$ & 23.31 & 23.31 & 85.96 & 40.95 & 3.48 & 0.89 & 63 & 11 & 0.98 & 0.93 \\
\hline & & St & 40.18 & 40.18 & 94.64 & 59.67 & 3.95 & 1.7 & 60 & 6 & 0.93 & 0.66 \\
\hline & & $\mathrm{Ch}$ & 82.6 & 82.6 & 95.63 & 97.01 & 4.4 & 3.6 & 172 & 32 & 0.96 & 0.98 \\
\hline & & $\mathrm{Ca}$ & 26.83 & 26.83 & 78.18 & 47.23 & 3.09 & 1.2 & 64 & 13 & 0.98 & 0.91 \\
\hline & & St & 43.64 & 43.64 & 91.82 & 69.3 & 3.95 & 1.76 & 62 & 7 & 0.81 & 0.54 \\
\hline & \multirow{3}{*}{ Body Movements } & $\mathrm{Ch}$ & 37.71 & 37.71 & 68.81 & 66.52 & 1.74 & 2.68 & 12 & 12 & 0.92 & 0.93 \\
\hline & & $\mathrm{Ca}$ & 32.67 & 32.67 & 58.71 & 56.79 & 2.64 & 1.34 & 33 & 15 & 0.85 & 0.88 \\
\hline & & St & 61.42 & 61.42 & 87.52 & 86.58 & 3.37 & 2.44 & 38 & 14 & 0.92 & 0.94 \\
\hline
\end{tabular}

Note: $\mathrm{Ch}=$ child $; \mathrm{Ca}=$ caregiver $; \mathrm{St}=$ stranger.

verbal expressions, and grammar of caregivers and strangers, while in children the levels of determinism, laminarity, and entropy tended to remain constant. In relation to the body movements displayed during the Strange Situation, adults showed slight increases in determinism levels and decreases in entropy and maximum line levels. In contrast, the percentage of determinism in children tended to remain stable, and entropy, especially in Video 1, tended to maintain its values, while in Video 2, a decrease was not as noticeable as in the case of adults.

Based on determinism and laminarity, it is possible to establish that communicative behaviours of caregivers and 
TABLE 5: Categorical CRQA measures for dyads in Videos 1 and 2.

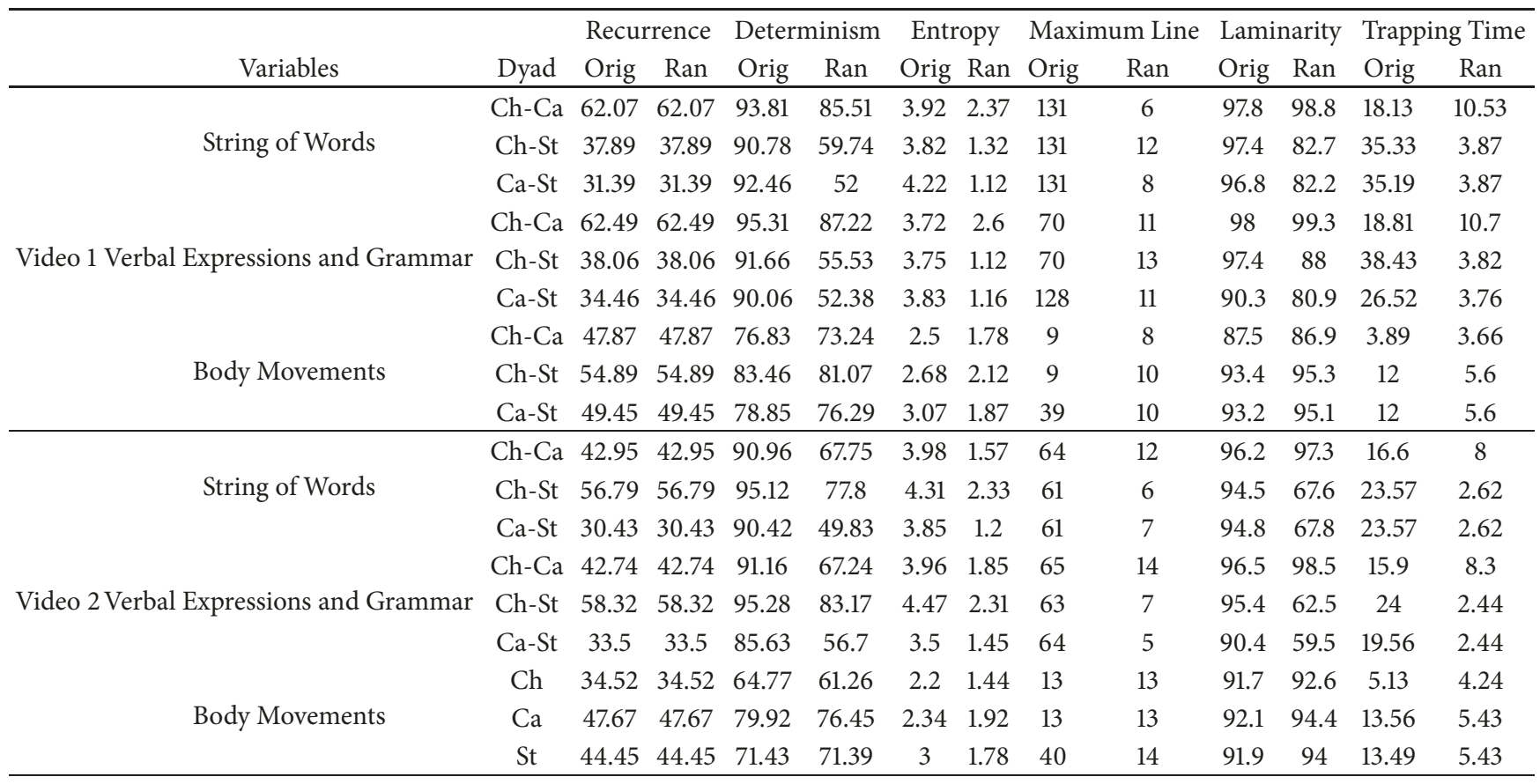

Note: $\mathrm{Ch}-\mathrm{Ca}=$ child-caregiver dyad; $\mathrm{Ch}-\mathrm{St}=$ child-stranger dyad; $\mathrm{Ca}-\mathrm{St}=$ caregiver-stranger dyad.

strangers had higher levels of synchronization with themselves compared with communicative behaviours of children. That is to say, the initial structure of verbal and motor behaviours was a strong predictor of subsequent behaviours. On the other hand, the decrease in entropy and maximum line indicated that there was a structure or pattern in the way behaviours were organized in natural conditions. And this pattern was different from a pattern of random organization.

\subsection{Categorical Cross-Recurrence Quantification Analysis for} Dyads in Videos 1 and 2. The results for dyads in Videos 1 and 2 (see Table 5) partially replicated the values observed with individuals. For string of words, verbal expressions, and grammar, the most notorious changes were observed at the level of determinism, entropy, maximum line, and trapping time, with the original time series having higher values than the randomized series. In contrast, for body movements, changes were detected in entropy and, to a lesser extent, in maximum line and laminarity. In the three dyads, the entropy levels were higher in the original series than in the randomized series, while only in the caregiver-stranger dyad the maximum line of the original series was greater than that of the randomized series. In verbal expressions, the dyads presented values that allow us to assume a degree of synchronization. However, in terms of motor behaviour, the caregiver-stranger dyad was the only one that showed slight signs of synchronization.

Our findings indicate at least three relevant aspects. Verbal and motor behaviours revealed different degrees of synchronization $[5,11]$. Words, verbal expressions, and grammar had more clear-cut indicators of synchronization and structure than body movements [43]. Likewise, individuals involved showed diverse degrees of synchronization with themselves. Both adults, caregiver and stranger, expressed better indicators of such internal coupling than infants. Finally, the synchronization indicators appeared clearly in all dyads, even when the caregiver-stranger dyad presented better indicators of coupling than the dyads where the infants were involved. The communicative interaction is a multidimensional phenomenon, in which a series of variables operating at different time scales are intertwined [44]. The analysis techniques used by us reduced the multidimensionality to the two dimensions present in the recurrence plot $[39,41,48]$. Verbal behaviours were expressed on a different time scale than motor behaviours. These verbal behaviours were a better example of the dynamic present in the Strange Situation. The dynamic was observed more clearly in the verbal behaviour of caregivers and strangers, especially when they were together in a dyad.

3.3. Comparison of Means between Groups Segmented by Communicative Behaviours, Individuals, and Dyads. Based on the recurrence measures obtained from both videos, we proceeded-in heuristic terms - to compare the original and randomized series with a Wilcoxon signed-rank test, a nonparametric test for related samples. The first comparison was segmenting by type of communicative behaviour (string of words, grammar, and body movements). In this case, the recurrence measures from individuals and dyads were grouped to estimate an average. The second comparison was among individuals (child, caregiver, and stranger). Finally, the third comparison was segmenting by dyads (childcaregiver, child-stranger, and caregiver-stranger). In both case, for individual and dyads, recurrence measures from 


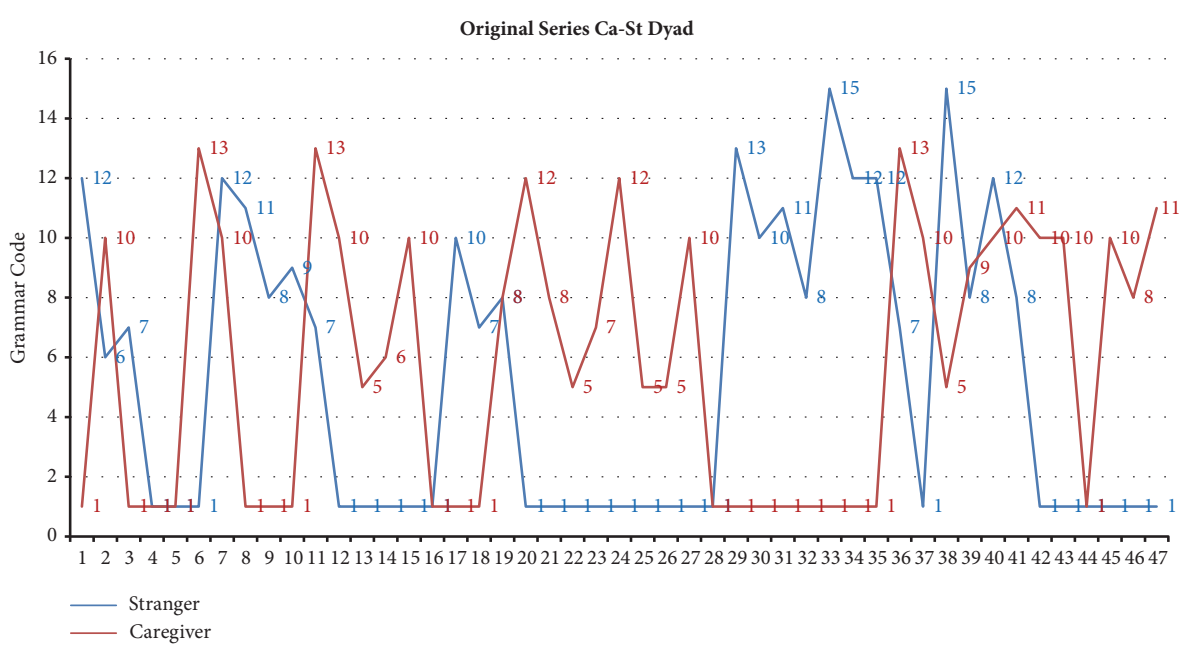

(a)

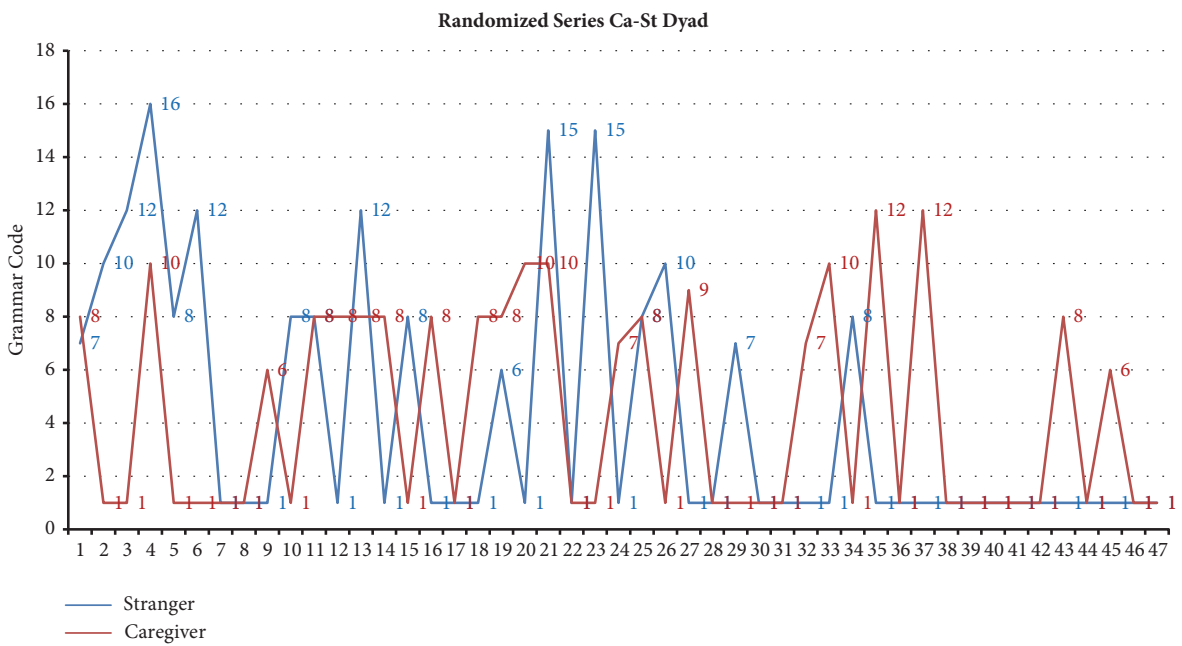

(b)

Figure 2: Panels (a) and (b) show the sequence of words that have been classified according to their grammatical function. In this segment of 47 events, only 12 categories appear. $1=$ silence, $5=\operatorname{article}, 6=$ pronoun, $7=$ noun, $8=$ verb, $9=$ adjective, $10=$ adverb, $11=$ conjunction, $12=$ preposition, 13 = interjection, $15=$ adverbial phrase, and $16=$ own name. Grammar observed in original series (Panel (a)) shows typical patterns of communicative interaction between caregiver and stranger. This typical pattern is cancelled when original time series are randomized (Panel (b)).

string of words, grammar and verbal expressions, and body movements were clustered to estimate their respective average.

As described in Figure 3 (Panels (a), (b), (c), and (d)), for the string of words the original series had a significantly higher percentage of determinism $(Z=-2.82, p=.005)$, entropy $(Z=-3.06, p=.002)$ and maximum line $(Z=-2.20$, $p=.028)$, and a marginally higher laminarity $(Z=-1.65, p$ $=.09)$ than in the randomized series. The same trend was observed with verbal expressions and grammar (Panels (e), (f), (g), and (f)), where the original series had significantly more determinism $(Z=-2.82, p=.005)$, entropy $(Z=-3.06$, $p=.002)$, and the maximum line $(Z=-3.06, p=.002)$ had a marginally higher laminarity $(Z=-1.65, p=.099)$ than the randomized series. For body movements (Panels (i), (j), (k), and (l)), the original series had higher levels of determinism
$(Z=-3.06, p=.002)$, entropy $(Z=-2.51, p=.012)$, maximum line $(Z=-2.32, p=.021)$, and laminarity $(Z=-2.43, p=.016)$ than the randomized series.

In Figure 4 (Panels (a), (b), (c), and (d)), segmenting by individuals, the children in the original series presented marginally higher levels of maximum line than in the randomized series $(Z=-1.83, p=.06)$; however no differences were detected in terms of determinism, entropy, and laminarity ( $Z s \leq-.11, p s \geq .91$ ). For caregivers (Panels (e), (f), (g), and (h)) and strangers (Panels (i), (j), (k), and (l)), the original series showed higher levels of determinism, entropy, and maximum line $(Z s \leq-2.20, p s \leq 0.028)$ than the randomized series. However, no differences were observed in terms of laminarity $(Z s \leq-1.58, p s \geq .11)$.

When focusing on dyads (Figure 5), it is possible to observe that the child-caregiver dyad (Panels (a), (b), (c), 


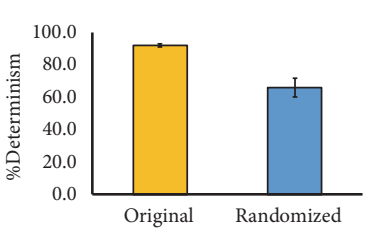

(a)

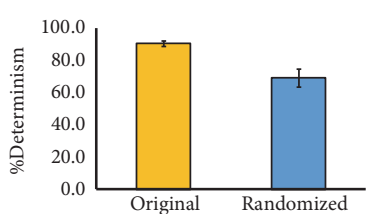

(e)

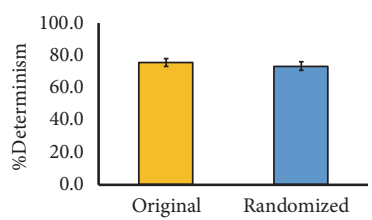

(i)

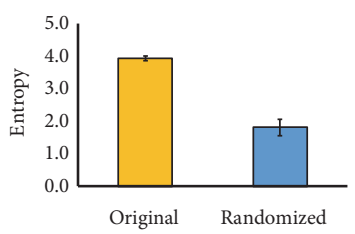

(b)

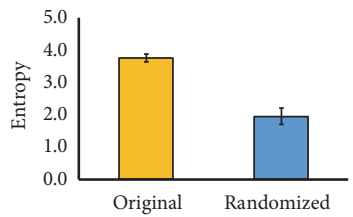

(f)

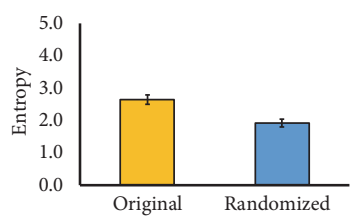

(j)

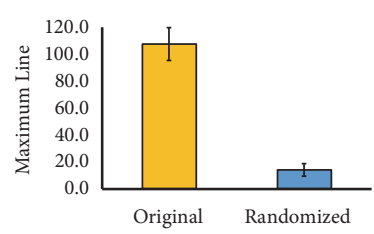

(c)

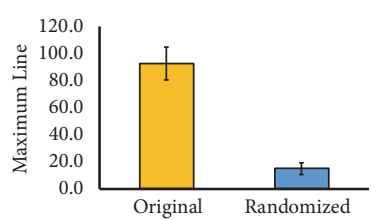

(g)

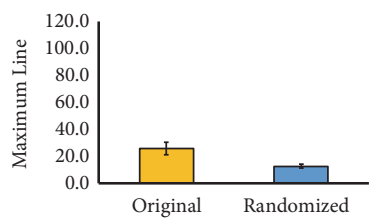

(k)

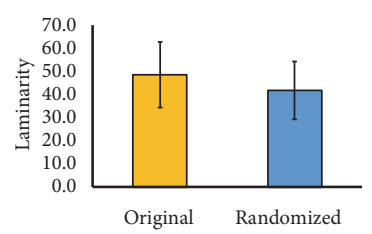

(d)

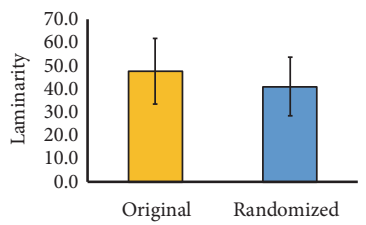

(h)

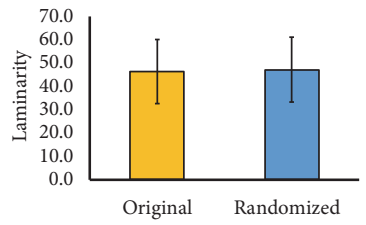

(1)

Figure 3: Mean and standard errors of determinism (Panels (a), (e), and (i)), entropy (Panels (b), (f), and (j)), maximum line (Panels (c), (g), and $(\mathrm{k})$ ), and laminarity (Panels (d), (h), and (l)), segmenting by words, verbal expressions and grammar, and body movements.

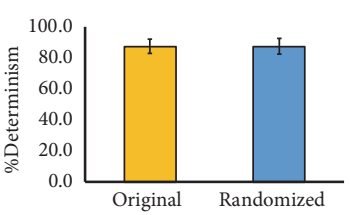

(a)

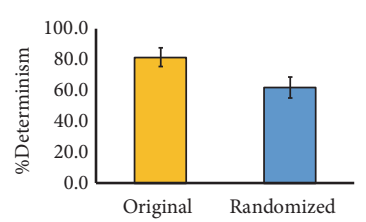

(e)

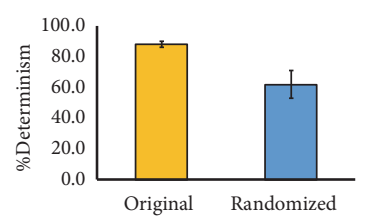

(i)

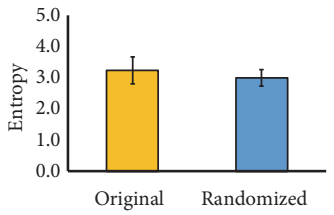

(b)

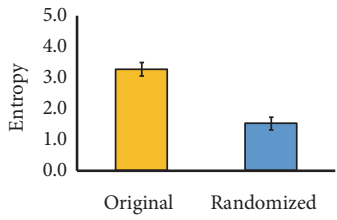

(f)

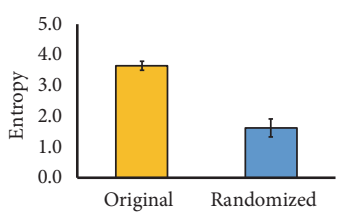

(j)

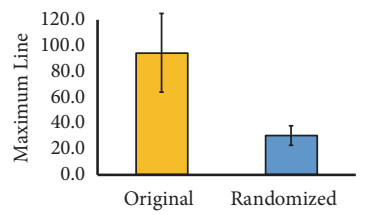

(c)

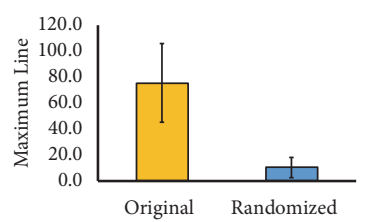

(g)

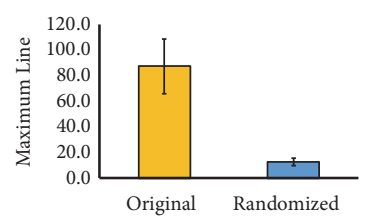

(k)

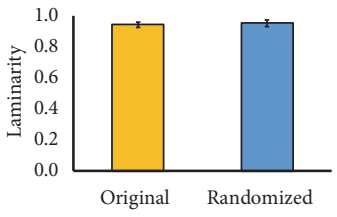

(d)

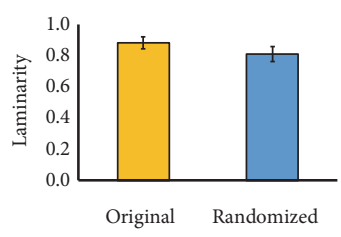

(h)

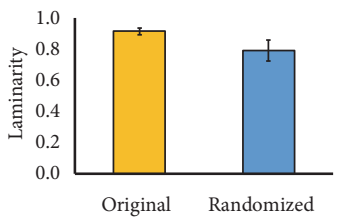

(l)

Figure 4: Mean and standard errors of determinism (Panels (a), (e), and (i)), entropy (Panels (b), (f), and (j)), maximum line (Panels (c), (g), and $(\mathrm{k})$ ), and laminarity (Panels (d), (h), and (l)) when segmenting by individuals (child, caregiver, and stranger).

(d), and (e)) in the original series had significantly more determinism, entropy, maximum line, and trapping time than in the randomized series $(Z s \geq-2.21$, $p s \leq .03)$, but no difference was observed in laminarity. The caregiverstranger dyad (Panels (l), (m), (n), (o), and (p)) and childstranger dyad (Panels (f), (g), (h), (i), and (j)) showed similar trends with the original series expressing higher levels of determinism, entropy, and trapping time $(Z s=-2.21, p s=$ $0.03)$, as well as a marginally higher maximum line $(Z=-1.75$, $p=0.08)$ than the randomized series. However, no differences were detected in laminarity $(Z=-1.51, p=.12)$.

With this nonparametric analysis, we corroborate what was previously reported from the visual inspection summarized in Tables 4 and 5. Words and verbal expressions 


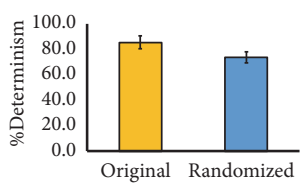

(a)

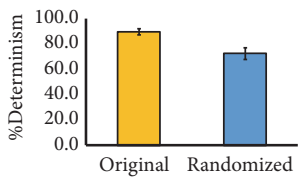

(f)

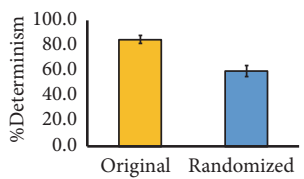

(k)

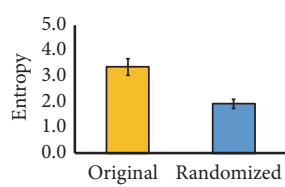

(b)

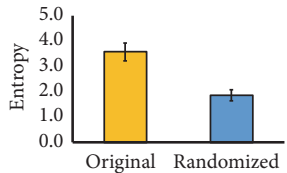

(g)

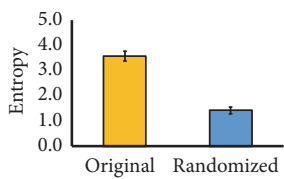

(1)

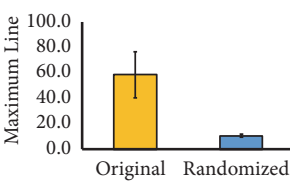

(c)

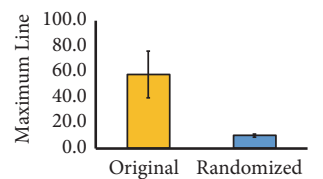

(h)

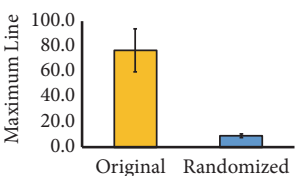

(m)

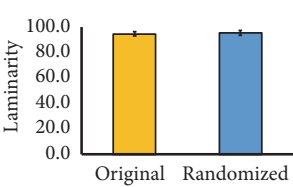

(d)

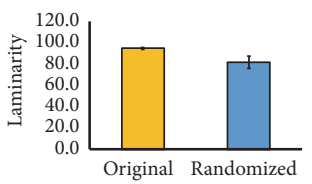

(i)

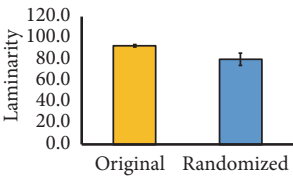

(n)

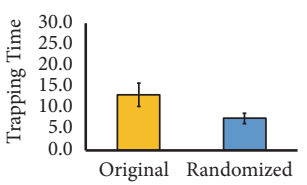

(e)

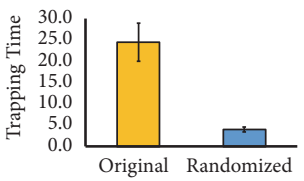

(j)

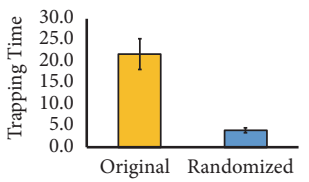

(o)

Figure 5: Mean and standard errors of determinism (Panels (a), (f), and (l)), entropy (Panels (b), (g), and (m)), maximum line (Panels (c), $(\mathrm{h})$, and (n)), laminarity (Panels (d), (i), and (o)), and trapping time (Panels (e), (j), and (p)) when segmenting by dyads (child-caregiver, child-stranger, and caregiver-stranger).

and grammar showed a structure that resembles a typical coupling pattern. This structure was more defined and clearer than the one observed in body movements. The actors involved in the Strange Situation showed noteworthy differences among them. While children clearly showed no traces of a structure, other than that observed by chance; the behaviours of caregivers and strangers had clear traces of dynamic patterns, typical of coupled systems. Finally, all dyads presented clear synchronization indicators, especially caregiver-child dyads. It is important to note that verbal and motor behaviours expressed by children did not have elements that indicate a coupling pattern. However, when these children interacted with their respective caregivers, the communicative behaviour between them clearly expressed signs of synchronization.

\section{Conclusions}

The purpose of this research was to characterize the degree of structuring of behaviours in order to identify the parameters of synchronization in a protocolized communicative interaction, Ainsworth's Strange Situation [21, 27, 31-33], by analysing the unfolded verbal and motor behaviours among caregivers, children, and strangers [31-33]. These behaviours were scrutinised using a nonlinear technique named Categorical Cross-Recurrence Quantification Analysis [5, 38, 39, 41-43]. From these analyses, it was expected to estimate measures that have been used to characterize degrees of coupling between systems [44-48].

The findings showed that words and verbal expressions and grammar had clear parameters of synchronization, taking into account the fact that determinism, entropy, maximum line, and laminarity were higher in the original series in comparison to the randomized series [16]. In the case of body movements, communicative patterns showed a type of synchronization with a recurrent structure, where the initial states enabled predicting the final states of the system, but whose stability was not different from a series where the motor behaviours appear in a random manner [40, 43-48, 51]. Thus, our results indicate that verbal behaviours - in the Strange Situation-are part of a communicative phenomenon that expresses higher levels of synchronization than motor behaviours $[45,46]$. This preliminary finding emphasizes that the communicative interaction has synchronization features, but these attributes are not homogeneous. If, until now, we assumed that communicative interaction-among three people interacting during a protocolized evaluation-was globally synchronized, our results suggest that some aspects have more dynamic characteristics than others.

For children, the values of determinism, entropy, maximum line, and laminarity remained constant between the original and randomized series. Thus, the structure of verbal and motor behaviours expressed for children was not different from what was to be expected if these behaviours appeared in a random manner. In contrast, for adults-caregivers or strangers - the values of determinism, entropy, laminarity, and maximum line were significantly reduced when their original series were randomized, suggesting that the original series of communicative behaviours had a synchronization pattern that was far from a random organization. This made us aware that, in a communicative interaction, not all actors involved have synchronized behaviours. However, when analysing the recurrence of two people interacting, the system itself shows traces of synchronization, even when one of the actors (in our case the children) does not show synchronization traits.

We are still blind to the attachment pattern of these two girls who participated in the Strange Situation. However, there are two possible scenarios that we conjecture. In the first one it can assumed that both infants have the same 
attachment pattern-regardless of whether this pattern is A, $\mathrm{B}, \mathrm{C}$, or $\mathrm{D}$-and therefore the observed values indicate a similar pattern of recurrence among them as observed in our study. Another possibility is that the attachments of these infants are different (generating the possible combinations of $\mathrm{A}$ and $\mathrm{B}, \mathrm{A}$ and $\mathrm{C}, \mathrm{A}$ and $\mathrm{D}, \mathrm{B}$ and $\mathrm{C}, \mathrm{B}$ and $\mathrm{D}$, or $\mathrm{C}$ and $D)$ [31-33]. Under this scenario, the observed recurrence values (entropy, laminarity, determinism, among others) did not detect the differences that the infants manifested in their attachment behaviours or how this pattern of attachment was unfolded in the interaction with adults. So, in order to disambiguate this problem our current work is aimed at analysing more Strange Situation videos, where each of the four types of attachment patterns can be represented proportionally. Thus, this preliminary investigation can be improved with the incorporation of more children classified according the four types of attachment [21], in such a quantity that comparisons can be made in terms more robust [44]. Attachment patterns are discrete categories defined by certain behaviours. Some of them promote communicative interactions with adults, while others restrict them [30]. Therefore, it is expected that recurrence parameters tend to vary from one type of attachment pattern to another.

Our study resembles a study with small samples or two unique cases study. The nonparametric contrasts that we conducted were purely heuristic and corroborated what the tables expressed. However, the possibility of comparing means depends on having an adequate sample size. Despite this limitation, the direct observation of the recurrence parameters in the recurrence plots is an extended practice, because the means and deviations are deceptive, insofar as they can hide the temporal structure of the behaviours [2, $8,40,43]$. Two groups could have the same mean and the same standard deviation and not show significant differences. However, they could have a different structure of variability over time. This last aspect is what the recurrence plots and RQA identify and that we have applied to Ainsworth's Strange Situation.

The Ainsworth's Strange Situation protocol is intended to study the infant's attachment pattern. In this protocol, two adults, the caregiver and the stranger, interact with the infant. However, it is also possible to observe that these adults interact with each other for a few minutes. In that sense, the interaction between the caregiver and the stranger is susceptible to be analysed in terms of verbal and motor behaviour. Considering that the central objective is to establish the attachment pattern of the infant, a rational and feasible decision could be to omit information regarding the few minutes of interaction between the caregiver and the stranger; however, we believe that it is early to make such a decision, considering that we are still estimating the weight that each actor has in the interactions that the protocol promotes. Social interactions have a dynamic character; therefore what will happen in the future is a function of what has happened in previous phases. Therefore, we suppose the interaction of these two adults in a previous phase could be an important ingredient that affects their interaction with the infant in the following phases.

\section{Data Availability}

The data used to support the findings of this study are available from the corresponding author upon request.

\section{Conflicts of Interest}

The authors declare that there are no conflicts of interest regarding the publication of this paper.

\section{Acknowledgments}

The authors thank Dr. José Luis Ulloa Fulgeri (Universidad de Talca, Chile), for his collaboration with the $\mathrm{R}$ program, and Dr. David López (University of Warsaw, Poland), for his suggestions and comments about preliminary version of this manuscript. This research has been funded by the Comisión Nacional de Investigación Cientifica y Tecnológica (CONICYT) under Research Grants REDES-170155 and PCIPAI80160101 to Ramón D. Castillo and by the Fondo Nacional de Desarrollo Científico y Tecnológico de Chile (FONDECYT) under Research Grant no. 1161533 to Ramón D. Castillo and Research Grant no. 1130773 to Rosario Spencer.

\section{Supplementary Materials}

The databases, coding matrices, and transcriptions of the interactions can be requested from Ramón D. Castillo (racastillo@utalca.cl) and (ramondanielcastillo@gmail.com). (Supplementary Materials)

\section{References}

[1] A. L. Goldberger, D. R. Rigney, and B. J. West, "Chaos and fractals in human physiology," Scientific American, vol. 262, no. 2, pp. 42-49, 1990.

[2] J. A. S. Kelso, Dynamic Patterns: The Self-Organization of Brain and Behavior, The MIT Press, Cambridge, MA, 1995.

[3] K. De Bot, W. Lowie, and M. Verspoor, "A dynamic systems theory approach to second language acquisition," Bilingualism: Language and Cognition, vol. 10, no. 1, pp. 7-21, 2007.

[4] M. M. Louwerse, R. Dale, E. G. Bard, and P. Jeuniaux, "Behavior matching in multimodal communication Is synchronized," Cognitive Science, vol. 36, no. 8, pp. 1404-1426, 2012.

[5] K. Shockley and M. Riley, "Interpersonal couplings in human interactions," in Recurrence quantifications analysis: Theory and best practices, C. Webber and N. Marwan, Eds., pp. 399-421, Springer, Heidelberg, Germany, 2015.

[6] D. L. Gilden, T. Thornton, and M. W. Mallon, "1/F Noise in human cognition," Science, vol. 267, no. 5205, pp. 1837-1839, 1995.

[7] D. L. Gilden, “Cognitive emissions of 1/f noise," Psychological Review, vol. 108, no. 1, pp. 33-56, 2001.

[8] M. A. Riley and M. T. Turvey, "Variability and determinism in motor behavior," Journal of Motor Behavior, vol. 34, no. 2, pp. 99-125, 2002.

[9] G. C. Van Orden, J. G. Holden, and M. T. Turvey, "Selforganization of cognitive performance," Journal of Experimental Psychology: General, vol. 132, no. 3, pp. 331-350, 2003. 
[10] C. T. Kello, B. C. Beltz, J. G. Holden, and G. C. Van Orden, "The Emergent Coordination of Cognitive Function," Journal of Experimental Psychology: General, vol. 136, no. 4, pp. 551-568, 2007.

[11] M. T. Turvey, "Action and perception at the level of synergies," Human Movement Science, vol. 26, no. 4, pp. 657-697, 2007.

[12] D. G. Stephen and D. Mirman, "Interactions dominate the dynamics of visual cognition," Cognition, vol. 115, no. 1, pp. 154$165,2010$.

[13] N. A. Kuznetsov and S. Wallot, "Effects of accuracy feedback on fractal characteristics of time estimation," Frontiers in Integrative Neuroscience, vol. 5, no. 62, pp. 1-12, 2011.

[14] C. A. Coey, M. Varlet, and M. J. Richardson, "Coordination dynamics in a socially situated nervous system," Frontiers in Human Neuroscience, vol. 6, no. 2012, pp. 1-12, 2012.

[15] M. Malone, R. D. Castillo, H. Kloos, J. G. Holden, M. J. Richardson, and R. Balasubramaniam, "Dynamic Structure of Joint-Action Stimulus-Response Activity," PLoS ONE, vol. 9, no. 2, p. e89032, 2014.

[16] R. D. Castillo, H. Kloos, J. G. Holden, and M. J. Richardson, "Long-range correlations and patterns of recurrence in children and adults' attention to hierarchical displays," Frontiers in Physiology, vol. 6, 2015.

[17] E. Delaherche, M. Chetouani, A. Mahdhaoui, C. Saint-Georges, S. Viaux, and D. Cohen, "Interpersonal synchrony: A survey of evaluation methods across disciplines," IEEE Transactions on Affective Computing, vol. 3, no. 3, pp. 349-365, 2012.

[18] R. Vink, M. L. Wijnants, A. H. N. Cillessen, and A. M. T. Bosman, "Cooperative learning and interpersonal synchrony," Nonlinear Dynamics, Psychology, and Life Sciences, vol. 21, no. 2, pp. 189-215, 2017.

[19] M. J. Hove and J. L. Risen, "It's all in the timing: Interpersonal synchrony increases affiliation," Social Cognition, vol. 27, no. 6, pp. 949-960, 2009.

[20] M. I. Coco and R. Dale, "Cross-recurrence quantification analysis of categorical and continuous time series: An R package," Frontiers in Psychology, vol. 5, article 510, 2014.

[21] M. Main and J. Solomon, "Procedures for identifying infants as disorganized/disoriented during the Ainsworth Strange Situation," in Attachment in the preschool years, M. T. Greenberg, D. Cicchetti, and E. M. Cummings, Eds., pp. 121-160, University of Chicago Press, Chicago, 1990.

[22] P. Fitzpatrick, V. Romero, J. L. Amaral et al., "Evaluating the importance of social motor synchronization and motor skill for understanding autism," Autism Research, vol. 10, no. 10, pp. 1687-1699, 2017.

[23] D. Stern, The interpersonal world of the infant, Basic Books, New York, NY, USA, 1985.

[24] M. Siller and M. Sigman, "The behaviors of parents of children with autism predict the subsequent development of their children's communication," Journal of Autism and Developmental Disorders, vol. 32, no. 2, pp. 77-89, 2002.

[25] B. Tunçgenç, E. Cohen, and C. Fawcett, "Rock With Me: The Role of Movement Synchrony in Infants' Social and Nonsocial Choices," Child Development, vol. 86, no. 3, pp. 976-984, 2015.

[26] J. Bowlby, "The nature of the child's tie to his mother," The International journal of psycho-analysis, vol. 39, no. 5, pp. 350373, 1958.

[27] J. Bowlby, A secure base. Clinical applications of Attachment Theory, Buenos Aires: Editorial Paidós, 1989.
[28] C. López and M. Ramírez, “Apego," Revista Chilena de Medicina Familiar, vol. 6, no. 1, pp. 20-24, 2005.

[29] A. Lichtwarck-Aschoff, F. Hasselman, R. Cox, D. Pepler, and I. Granic, "A characteristic destabilization profile in parent-child interactions associated with treatment efficacy for aggressive children," Nonlinear Dynamics, Psychology, and Life Sciences, vol. 16, no. 3, pp. 353-379, 2012.

[30] B. E. Vaughn, S. Goldberg, L. Atkinson, S. Marcovitch, D. MacGregor, and R. Seifer, "Quality of Toddler-Mother Attachment in Children with Down Syndrome: Limits to Interpretation of Strange Situation Behavior," Child Development, vol. 65, no. 1, pp. 95-108, 1994.

[31] M. D. S. Ainsworth, Infancy in Uganda: infant care and growth of attachment, University Press, Baltimore, Maryland: Johns Hopkins, 1967.

[32] M. D. S. Ainsworth, M. C. Blehar, E. Waters, and S. Wall, Patterns of attachment: A Psychological Study of Strange Situation, Lawrence Erlbaum, Hillsdale, NJ, USA, 1978.

[33] M. D. Ainsworth and S. M. Bell, "Attachment, exploration, and separation: illustrated by the behavior of one-year-olds in a strange situation.," Child Development, vol. 41, no. 1, pp. 49-67, 1970.

[34] C. Beckner, R. Blythe, J. Bybee et al., "Language is a complex adaptive system: Position paper," Language Learning, vol. 59, no. 1, pp. 1-26, 2009.

[35] S. Wallot, "Recurrence Quantification Analysis of Processes and Products of Discourse: A Tutorial in R," Discourse Processes, vol. 54, no. 5-6, pp. 382-405, 2017.

[36] D. H. Abney, A. S. Warlaumont, A. Haussman, J. M. Ross, and S. Wallot, "Using nonlinear methods to quantify changes in infant limb movements and vocalizations," Frontiers in Psychology, vol. 5, p. 771, 2014.

[37] F. Ramseyer and W. Tschacher, "Nonverbal synchrony of headand body-movement in psychotherapy: Different signals have different associations with outcome," Frontiers in Psychology, vol. 979, no. 5, pp. 1-9, 2014.

[38] J. P. Zbilut and C. L. Webber Jr., "Embeddings and delays as derived from quantification of recurrence plots," Physics Letters A, vol. 171, no. 3-4, pp. 199-203, 1992.

[39] N. Marwan, M. Carmen Romano, M. Thiel, and J. Kurths, "Recurrence plots for the analysis of complex systems," Physics Reports, vol. 438, no. 5-6, pp. 237-329, 2007.

[40] F. Orsucci, A. Giuliani, C. Webber Jr., J. Zbilut, P. Fonagy, and M. Mazza, "Combinatorics and synchronization in natural semiotics," Physica A: Statistical Mechanics and its Applications, vol. 361, no. 2, pp. 665-676, 2006.

[41] K. Shockley, "Cross recurrence quantification of interpersonal postural activity," in Tutorials in contemporary nonlinear methods for the behavioral sciences, M. A. Riley and G. C. Van Orden, Eds., pp. 142-177, 2005, https://www.nsf.gov/sbe/bcs/pac/nmbs/chap4.psd.

[42] M. A. Riley, R. Balasubramaniam, and M. T. Turvey, "Recurrence quantification analysis of postural fluctuations," Gait \& Posture, vol. 9, no. 1, pp. 65-78, 1999.

[43] C. L. Webber and J. P. Zbilut, "Recurrence Quantification Analysis of Nonlinear Dynamical Systems," in Tutorials in contemporary nonlinear methods for the behavioral sciences, $\mathrm{M}$. A. Riley and G. C. Van Orden, Eds., vol. 94, pp. 26-94, 2005, https://www.nsf.gov/sbe/bcs/pac/nmbs/chap4.psd.

[44] D. C. Richardson and R. Dale, "Looking To Understand: The Coupling Between Speakers' and Listeners' Eye Movements 
and Its Relationship to Discourse Comprehension," Cognitive Science, vol. 29, no. 6, pp. 1045-1060, 2005.

[45] M. J. Spivey and R. Dale, "Continuous dynamics in real-time cognition," Current Directions in Psychological Science, vol. 15, no. 5, pp. 207-211, 2006.

[46] D. C. Richardson, R. Dale, and N. Z. Kirkham, "The Art of Conversation Is Coordination," Psychological Science, vol. 18, no. 5, pp. 407-413, 2007.

[47] R. F. A. Cox and M. van Dijk, "Microdevelopment in ParentChild Conversations: From Global Changes to Flexibility," Ecological Psychology Journal, vol. 25, no. 3, pp. 304-315, 2013.

[48] J. P. Zbilut, A. Giuliani, and C. L. Webber Jr., "Detecting deterministic signals in exceptionally noisy environments using cross-recurrence quantification," Physics Letters A, vol. 246, no. 1-2, pp. 122-128, 1998.

[49] R. Fusaroli, I. Konvalinka, and S. Wallot, "Analyzing Social Interactions: The Promises and Challenges of Using Cross Recurrence Quantification Analysis," in Translational Recurrences: From Mathematical Theory to Real-World Applications, N. Marwan, M. Riley, A. Giuliani, and C. Webber, Eds., vol. 103, pp. 137-155, Springer International Publishing, London, UK, 2014.

[50] L. De Jonge-Hoekstra, S. Van der Steen, P. Van Geert, and R. F. Cox, "Asymmetric Dynamic Attunement of Speech and Gestures in the Construction of Children's Understanding," Frontiers in Psychology, vol. 7, 2016.

[51] R. Dale and M. J. Spivey, "Unraveling the dyad: Using recurrence analysis to explore patterns of syntactic coordination between children and caregivers in conversation," Language Learning, vol. 56, no. 3, pp. 391-430, 2006.

[52] A. Warlaumont, D. Oller, and R. Dale, "Vocal interaction dynamics of children with and without autism," in Proceedings of the 32nd Annual Meeting of the Cognitive Science Society, S. Ohlsson and R. Catrambone, Eds., pp. 121-126, Cognitive Science Society, Austin, TX, 2010.

[53] S. Wallot, B. A. O’Brien, A. Haussmann, H. Kloos, and M. S. Lyby, "The role of reading time complexity and reading speed in text comprehension," Journal of Experimental Psychology: Learning, Memory, and Cognition, vol. 40, no. 6, pp. 1745-1765, 2014.

[54] R. Schmidt and M. Richardson, "Dynamics of interpersonal coordination," in Coordination: Neural, Behavioral and Social Dynamics, Fuch and V. Jirsa, Eds., pp. 281-308, Springer-Verlag, Heidelberg, Germany, 2008.

[55] J. A. De Graag, R. F. A. Cox, F. Hasselman, J. Jansen, and C. De Weerth, "Functioning within a relationship: Mother-infant synchrony and infant sleep," Infant Behavior \& Development, vol. 35, no. 2, pp. 252-263, 2012.

[56] J. G. Holden, I. Choi, P. G. Amazeen, and G. Van Orden, "Fractal 1/ $f$ Dynamics Suggest Entanglement of Measurement and Human Performance," Journal of Experimental Psychology: Human Perception and Performance, vol. 37, no. 3, pp. 935-948, 2011.

[57] J. G. Holden, G. C. Van Orden, and M. T. Turvey, "Dispersion of Response Times Reveals Cognitive Dynamics," Psychological Review, vol. 116, no. 2, pp. 318-342, 2009.

[58] C. T. Kello, G. G. Anderson, J. G. Holden, and G. C. Van Orden, "The pervasiveness of $1 / \mathrm{f}$ scaling in speech reflects the metastable basis of cognition," Cognitive Science, vol. 32, no. 7, pp. 1217-1231, 2008.
[59] E.-J. Wagenmakers, H. L. J. Van Der Maas, and S. Farrell, "Abstract Concepts Require Concrete Models: Why Cognitive Scientists Have Not Yet Embraced Nonlinearly Coupled, Dynamical, Self-Organized Critical, Synergistic, Scale-Free, Exquisitely Context-Sensitive, Interaction-Dominant, Multifractal, Interdependent Brain-Body-Niche Systems," Topics in Cognitive Science, vol. 4, no. 1, pp. 87-93, 2012.

[60] E.-J. Wagenmakers, S. Farrell, and R. Ratcliff, "Human cognition and a pile of sand: A discussion on serial correlations and self-organized criticality," Journal of Experimental Psychology: General, vol. 134, no. 1, pp. 108-116, 2005.

[61] D. L. Gilden, "Global model analysis of cognitive variability," Cognitive Science, vol. 33, no. 8, pp. 1441-1467, 2009. 


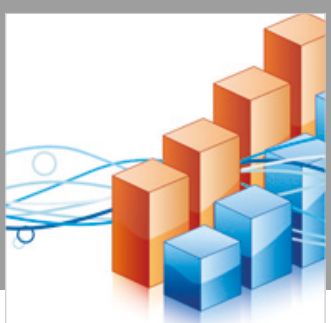

Advances in

Operations Research

\section{-n-m}
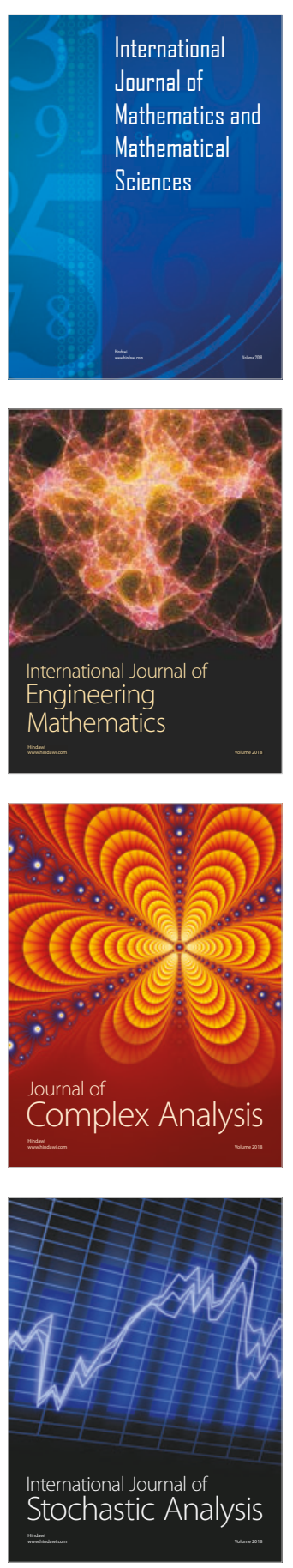
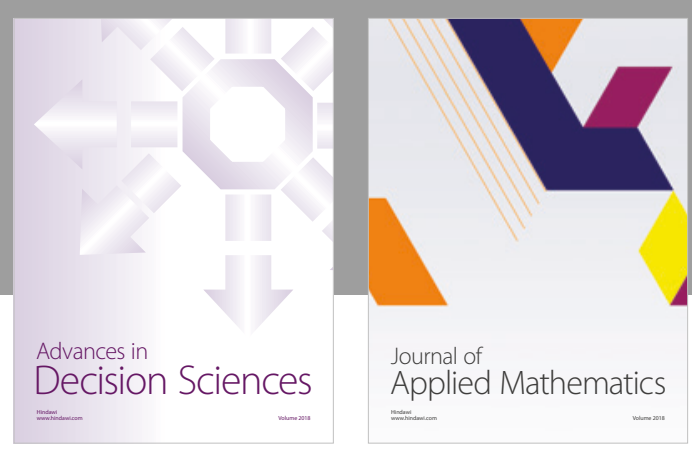

Journal of

Applied Mathematics
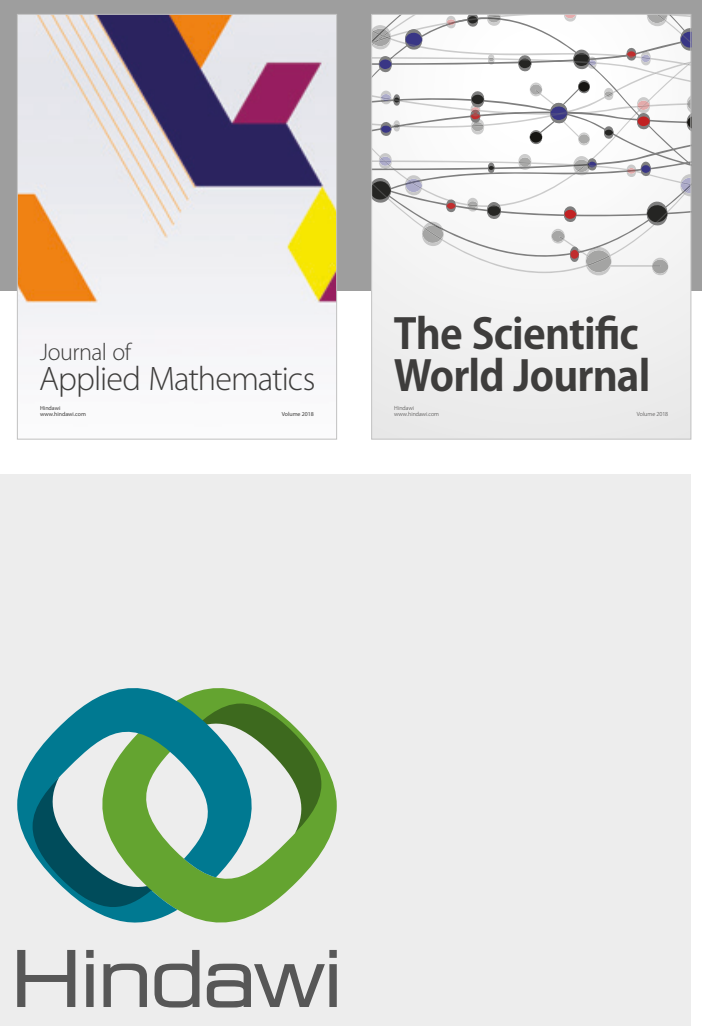

Submit your manuscripts at

www.hindawi.com

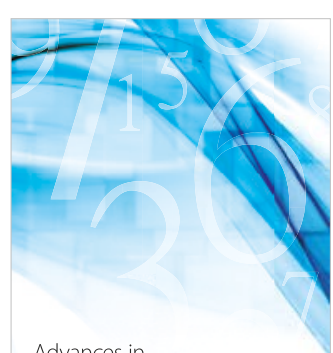

Advances in
Numerical Analysis
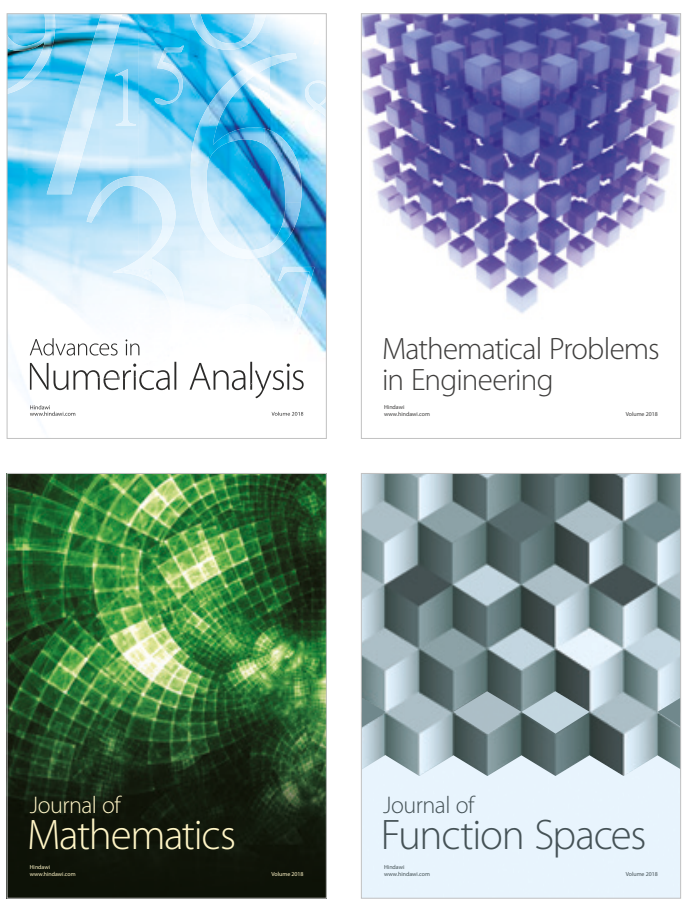

Mathematical Problems in Engineering

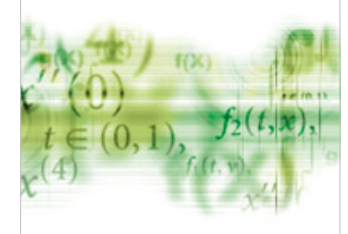

International Journal of

Differential Equations

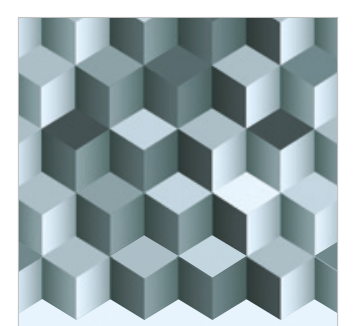

Journal of

Function Spaces
The Scientific

World Journal

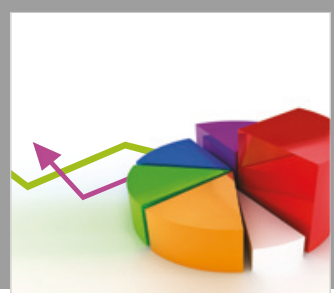

Journal of

Probability and Statistics
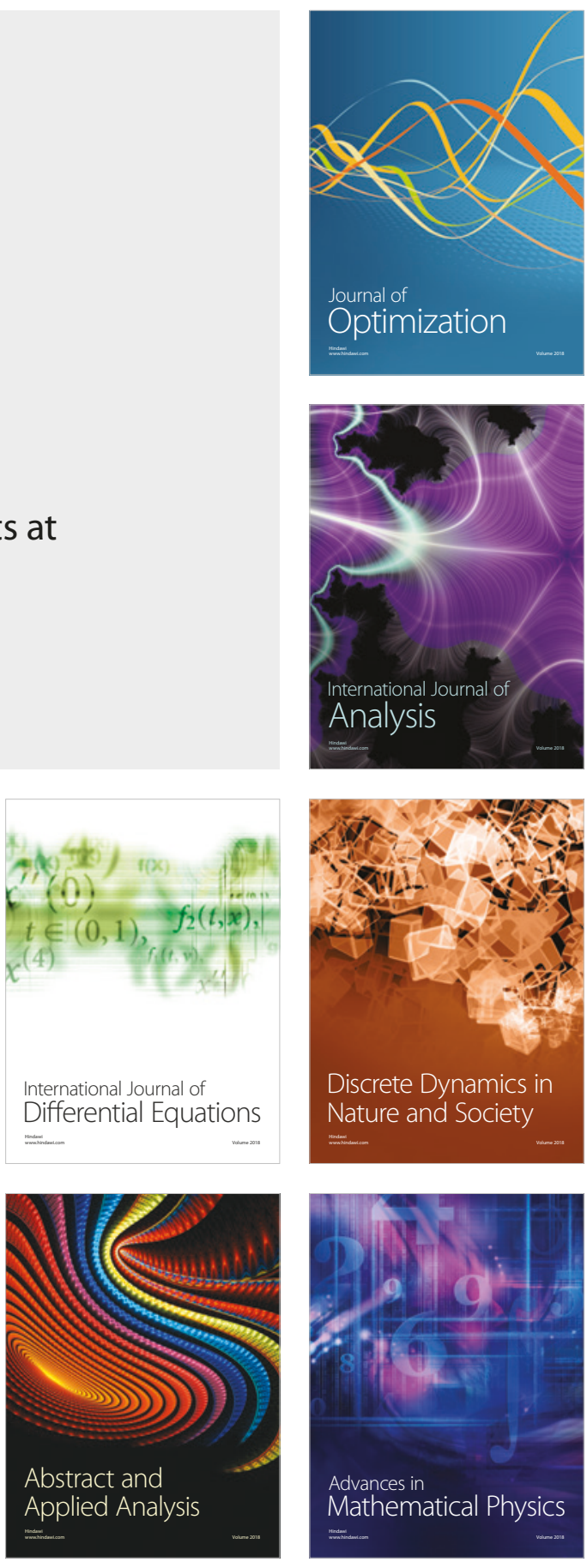